\title{
Supercontinuum comb sources for broadband communications based on AIGaAs-on- insulator
}

Hu, Hao; Pu, Minhao; Da Ros, Francesco; Galili, Michael; Yvind, Kresten; Morioka, Toshio; Oxenløwe, Leif Katsuo

\section{Published in:}

Proceedings of SPIE

Link to article, DOI:

$10.1117 / 12.2256032$

Publication date:

2017

Document Version

Publisher's PDF, also known as Version of record

Link back to DTU Orbit

Citation $(A P A)$ :

Hu, H., Pu, M., Da Ros, F., Galili, M., Yvind, K., Morioka, T., \& Oxenløwe, L. K. (2017). Supercontinuum comb sources for broadband communications based on AIGaAs-on-insulator. In Proceedings of SPIE (Vol. 10088). [100880C] SPIE - International Society for Optical Engineering. Proceedings of SPIE - The International Society for Optical Engineering https://doi.org/10.1117/12.2256032

\section{General rights}

Copyright and moral rights for the publications made accessible in the public portal are retained by the authors and/or other copyright owners and it is a condition of accessing publications that users recognise and abide by the legal requirements associated with these rights.

- Users may download and print one copy of any publication from the public portal for the purpose of private study or research.

- You may not further distribute the material or use it for any profit-making activity or commercial gain

- You may freely distribute the URL identifying the publication in the public portal 


\title{
Supercontinuum comb sources for broadband communications based on AlGaAs-on-insulator
}

\author{
Hao Hu*, Minhao Pu, Francesco Da Ros, Michael Galili, Kresten Yvind, Toshio Morioka, \\ Leif K. Oxenløwe \\ DTU Fotonik, Department of Photonics Engineering, Technical University of Denmark, DK-2800, \\ Lyngby, Denmark.
}

\begin{abstract}
We experimentally demonstrated $10 \mathrm{GHz}$ frequency comb spectral broadening in an AlGaAsOI nano-waveguide with the peak power of only several watts. The spectral broadened $10 \mathrm{GHz}$ frequency comb has high optical signal to noise ratio (OSNR) at the output of the nano-waveguide. As far as we know, it is the first photonic chip based frequency comb, relying on spectral broadening of a $10 \mathrm{GHz}$ mode-locked laser comb in an AlGaAsOI nano-waveguide, with a sufficient comb output power to support several hundred Tbit/s optical data.
\end{abstract}

Keywords: Supercontinuum generation, fiber optics communications, nonlinear optics, integrated optics, waveguide

\section{INTRODUCTION}

As cost and energy consumption are becoming limiting factors in high-capacity systems, using fewer lasers with less energy consumption grows desirable and demonstrations of ultra-high-capacity optical transmission based on nonlinear fiber based spectral broadening of a seed laser frequency comb have been reported ${ }^{1,2,3,4}$. The prospect of moving the fiber-based frequency comb sources onto a photonic chip platform holds many attractive features, including ultra-high bandwidth, stable polarization and phase, absence of stimulated Brillouin scattering (SBS), and the possibility of photonic integration with a seed laser ${ }^{5}$.

AlGaAs-on-insulator (AlGaAsOI) has shown to be an ultra-efficient nonlinear material platform ${ }^{6,7}{ }^{\text {. It combines high }}$ intrinsic material nonlinearity (on the order of $10^{-17} \mathrm{~W} / \mathrm{m}^{2}$ ), large index contrast between AlGaAs (3.3) and silica cladding (1.5), and low linear and nonlinear losses. The bandgap of AlGaAs can also be engineered by changing the $\mathrm{Al}$ concentration to avoid two-photon absorption (TPA) in the telecom wavelengths.

In this paper, we present the photonic chip based frequency comb, relying on spectral broadening of a mode-locked laser comb in an AlGaAsOI nano-waveguide, with a sufficient comb output power to support several hundred Tbit/s optical data ${ }^{9}$. We use a $33.6 \mathrm{~nm}$ wide part of the generated comb spectrum to carry $80 \times 40$ Gbaud WDM channels with PDM16-QAM modulation format. The high comb OSNR allows us to send the signal over 30 spatial channels, and we demonstrate successful $9.6 \mathrm{~km}$ transmission in a heterogeneous 30-core fiber reaching a total of $661 \mathrm{Tbit} / \mathrm{s}$ after FEC overhead subtraction.

\section{SPECTRAL BROADENING OF A 10 GHZ MODE-LOCKED LASER COMB}

Figure 1 shows the experimental setup for frequency comb spectral broadening in an AlGaAsOI nano-waveguide. An erbium glass oscillator (ERGO) generates a $10 \mathrm{GHz}$ pulse train at $1550 \mathrm{~nm}$ with picosecond pulse width. The $10 \mathrm{GHz}$ modulation frequency is locked to a microwave oscillator with an accuracy of $\sim \mathrm{Hz}$ and the absolute frequency of the pulse can be fine-tuned without the need of external reference laser. After amplification in an erbium-doped fiber amplifier (EDFA), the pulses are launched into the AlGaAsOI nano-waveguide. A polarization controller is used to align the pulses to the transverse electric (TE) mode of the waveguide and an optical spectrum analyzer is used to record the output spectrum.

*huhao@fotonik.dtu.dk; phone +45 45253783; fax +45 45936581; www.fotonik.dtu.dk

Nonlinear Frequency Generation and Conversion: Materials and Devices XVI, edited by

Konstantin L. Vodopyanov, Kenneth L. Schepler, Proc. of SPIE Vol. 10088, 100880C

(C) 2017 SPIE · CCC code: 0277-786X/17/\$18 - doi: 10.1117/12.2256032 


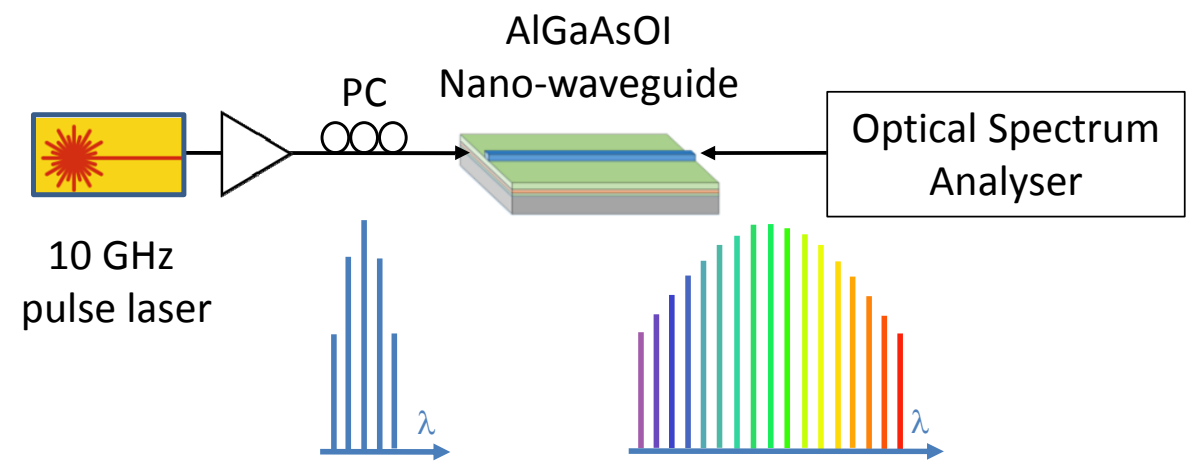

Figure 1. Experimental setup for frequency comb spectral broadening using an AlGaAsOI nano-waveguide.

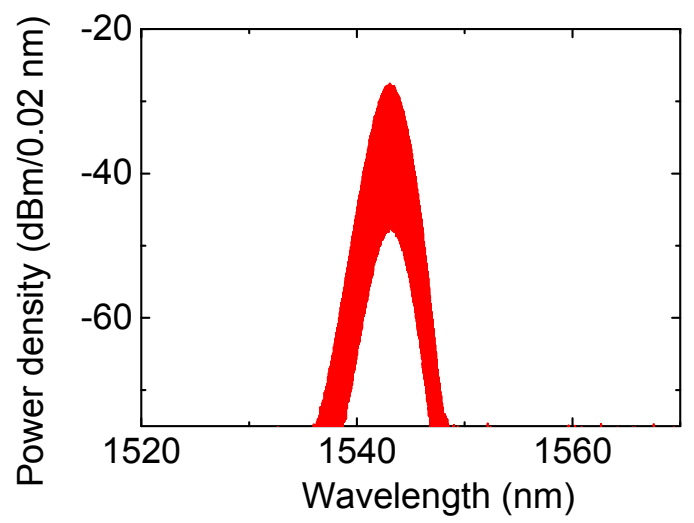

(a)

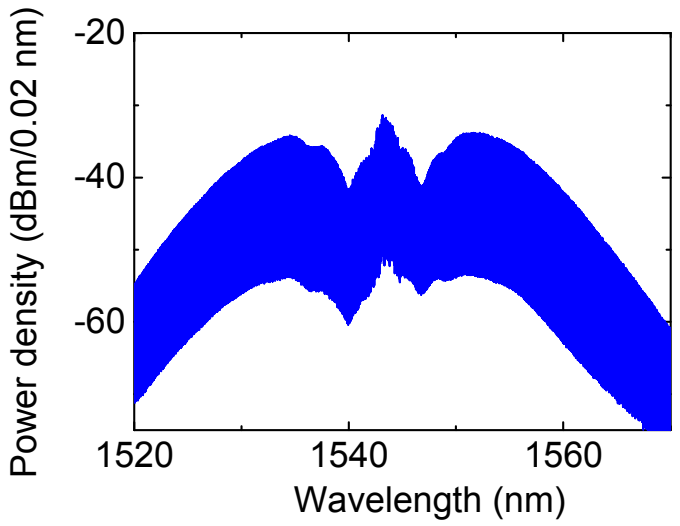

(b)

Figure 2. Optical spectra of $10 \mathrm{GH}$ mode-locked laser frequency comb (a) and spectrally broadened frequency comb at the output of the AlGaAsOI nano-waveguide (b).

Figure 2 (a) shows the spectrum of the ps pump pulse at the input of AlGaAsOI nano-waveguide (Fig. 3(a)) and Figure 2 (b) shows the spectrum at the output of the waveguide with an average launched power of $19.3 \mathrm{dBm}$ (peak power of $\sim 5.6$ W). The broadened spectrum at the output of the AlGaAsOI nano-waveguide has a $20-\mathrm{dB}$ bandwidth of $\sim 44 \mathrm{~nm}$, which is used as the wavelength division multiplexed (WDM) light source.

\section{SINGLE SOURCE OPTICAL TRANSMISSION USING THE SPECTRALLY BROADENED FREQUENCY COMB}

Figure 3 (c) shows the experimental setup of single source optical data transmission using the spectrally broadened frequency comb. The single source laser in the transmitter is an Erbium glass oscillating mode-locked laser, which produces 10-GHz pulses (1542 nm, 1.5-ps FWHM). The pulses are amplified and used to generate an optical frequency comb based on self-phase modulation (SPM) in the AlGaAsOI photonic chip (Fig. 3 (b)).

The estimated OSNR is $\sim 43 \mathrm{~dB}$ at $1552 \mathrm{~nm}$ and $\sim 30 \mathrm{~dB}$ at $1563 \mathrm{~nm}$. The broadened spectrum is equalized in a wavelength selective switch (WSS) and the inhomogeneous part in the center is replaced by the original spectrum from the mode-locked laser through another path, which results in a flat and stable frequency comb ${ }^{1}$. The equalized frequency comb works as the light sources for $80 \mathrm{WDM}$ channels with $50 \mathrm{GHz}$ spacing. For each WDM channel, the signal is modulated with PDM-16-QAM and then OTDM multiplexed to 40 Gbaud. The signal is transmitted through the 9.6-km 30 -core single-mode fiber, with a total data capacity of $661 \mathrm{Tbit} / \mathrm{s}^{9}$. 


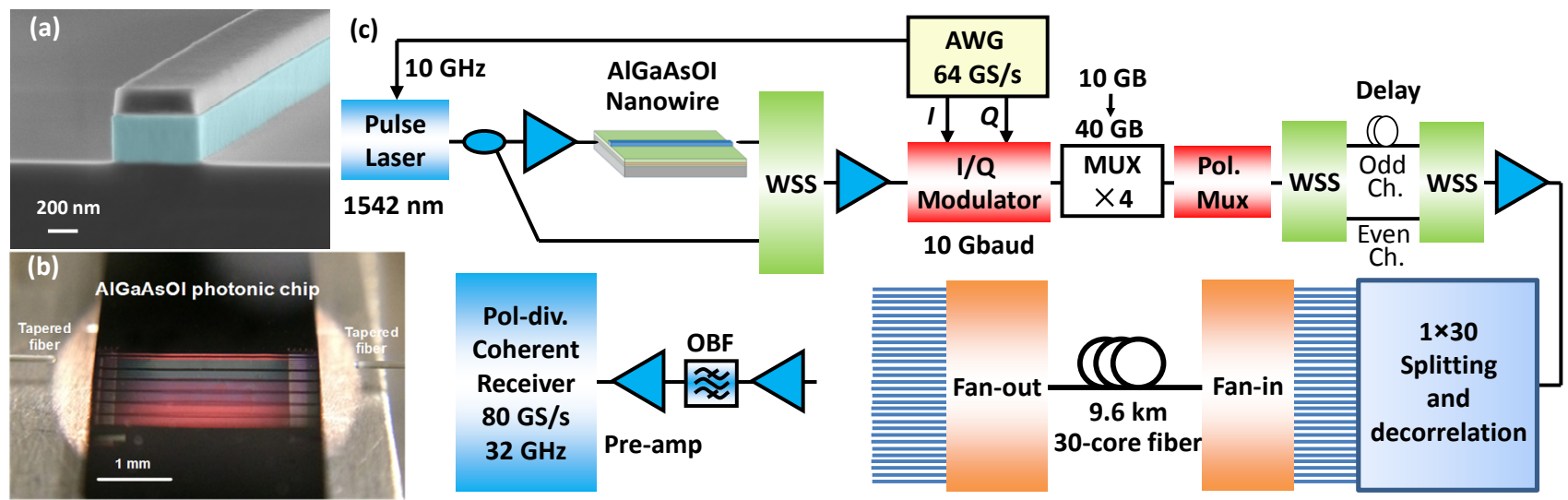

Figure 3. (a) SEM picture of a fabricated AlGaAsOI nano-waveguide (denoted by the artificial blue color). (b) Photograph of the AlGaAsOI photonic chip. (c) Schematic of the experimental setup for the $661 \mathrm{Tbit} / \mathrm{s}$ single-source AlGaAs frequency comb transmitter for 30-core transmission demonstration.

\section{CONCLUSION}

We have demonstrated $10 \mathrm{GHz}$ spectrally broadened frequency comb based on an AlGaAsOI nano-waveguide, which has a $20-\mathrm{dB}$ bandwidth of $\sim 44 \mathrm{~nm}$ and cover the telecom $\mathrm{C}$ band. The spectrally broadened $10 \mathrm{GHz}$ frequency comb has enough OSNR to carry $661 \mathrm{Tbit} / \mathrm{s}$ data in a fully loaded 30-core fiber. This is the highest reported amount of data carried on the light generated from a chip-based single-source transmitter, and we demonstrate successful $9.6 \mathrm{~km}$ transmission.

\section{REFERENCES}

[1] D. Hillerkuss, R. Schmogrow, T. Schellinger, M. Jordan, M. Winter, G. Huber, T. Vallaitis, R. Bonk, P. Kleinow, F. Frey, M. Roeger, S. Koenig, A. Ludwig, A. Marculescu, J. Li, M. Hoh, M. Dreschmann, J. Meyer, S.B. Ezra, N. Narkiss, B. Nebendahl, F. Parmigiani, P. Petropoulos, B. Resan, A. Oehler, K. Weingarten, T. Ellermeyer, J. Lutz, M. Moeller, M. Huebner, J. Becker, C. Koos, W. Freude, J. Leuthold, " 26 Tbit s-1 line-rate super-channel transmission utilizing all-optical fast Fourier transform processing," Nat Photon 5, 364-371 (2011).

[2] V. Ataie, E. Temprana, L. Liu, Y. Myslivets, P. P. Kuo, N. Alic, and S. Radic, "Flex-grid Compatible Ultra Wide Frequency Comb Source for $31.8 \mathrm{~Tb} / \mathrm{s}$ Coherent Transmission of 1520 UDWDM Channels," in Optical Fiber Communication Conference: Postdeadline Papers, (Optical Society of America, 2014), paper Th5B.7 (2014).

[3] H. Hu, F. Ye, A. K. Medhin, P. Guan, H. Takara, Y. Miyamoto, H. C. H. Mulvad, M. Galili, T. Morioka, and L. K. Oxenlowe, "Single Source 5-dimensional (Space-, Wavelength-, Time-, Polarization-, Quadrature-) 43 Tbit/s Data Transmission of 6 SDM \&times; 6 WDM \&times; 1.2 Tbit/s Nyquist-OTDM-PDM-QPSK," in CLEO: 2014 Postdeadline Paper Digest, OSA Technical Digest (online) (Optical Society of America, 2014), paper JTh5B.10 (2104).

[4] B. J. Puttnam, R. S. Luís, W. Klaus, J. Sakaguchi, J.-M. Delgado Mendinueta, Y. Awaji, N. Wada, Y. Tamura, T. Hayashi, M. Hirano, and J. Marciante, " $2.15 \mathrm{~Pb} / \mathrm{s}$ transmission using a 22 core homogeneous single-mode multi-core fiber and wideband optical comb," in Proc. ECOC, PDP.3.1 (2015).

[5] J. Pfeifle, A. Kordts, P. Marin, M. Karpov, M. Pfeiffer, V. Brasch, R. Rosenberger, J. Kemal, S. Wolf, W. Freude, t. kippenberg, and C. Koos, "Full C and L-Band Transmission at $20 \mathrm{Tbit} / \mathrm{s}$ Using Cavity-Soliton Kerr Frequency Combs," in CLEO: 2015 Postdeadline Paper Digest, (Optical Society of America, 2015), paper JTh5C.8 (2015).

[6] M. Pu, H. Hu, L. Ottaviano, E. Semenova, D. Vukovic, L. K. Oxenlowe, and K. Yvind, "AlGaAs-On-Insulator Nanowire with $750 \mathrm{~nm}$ FWM Bandwidth, $-9 \mathrm{~dB}$ CW Conversion Efficiency, and Ultrafast Operation Enabling Record Tbaud Wavelength Conversion," in Optical Fiber Communication Conference Post Deadline Papers, OSA Technical Digest (online) (Optical Society of America, 2015), paper Th5A.3 (2015). 
[7] Luisa Ottaviano, Minhao Pu, Elizaveta Semenova, and Kresten Yvind, "Low-loss high-confinement waveguides and microring resonators in AlGaAs-on-insulator," Opt. Lett. 41, 3996-3999 (2016).

[8] Minhao Pu, Luisa Ottaviano, Elizaveta Semenova, and Kresten Yvind, "Efficient frequency comb generation in AlGaAs-on-insulator," Optica 3, 823-826 (2016).

[9] H. Hu, F. Da Ros, F. Ye, M. Pu, K. Ingerslev, E. Porto da Silva, M. Nooruzzaman, Y. Amma, Y. Sasaki, T. Mizuno, Y. Miyamoto, L. Ottaviano, E. Semenova, P. Guan, D. Zibar, M. Galili, K. Yvind, L. K. Oxenløwe, and T. Morioka, "Single-Source AlGaAs Frequency Comb Transmitter for 661 Tbit/s Data Transmission in a 30-core Fiber," in Conference on Lasers and Electro-Optics, OSA Technical Digest (online) (Optical Society of America, 2016), paper JTh4C.1 (2016). 